\title{
A Fuzzy Negotiation Model with Genetic Algorithms
}

\author{
Dongsheng Zhai, Yuying Wu, Jinxuan Lu, Feng Yan \\ School of Economics and Management, Beijing University of Technology, \\ Beijing, China, 100022 \\ zhaidongsheng@bjut.edu.cn hlbao@bjut.edu.cn \\ lvjinxuan@emails.bjut.edu.cn yanfeng@bjut.edu.cn
}

\begin{abstract}
An offer in a fuzzy negotiation model is rejected or accepted by acceptability based on fuzzy set theory and membership functions. Since different issues have different effect on negotiators, the combined concession in the multi-issue negotiation for negotiators and negotiation agents and genetic learning mechanism are adopted to update their beliefs about incomplete information. The fuzzy negotiation model with genetic algorithms is more practical than the traditional negotiation model.
\end{abstract}

\section{Introduction}

Automatic negotiation and transaction on the internet is becoming more popular with the development of computer technology and e-commerce, and more and more people accept it as a new way of doing business. The negotiation and transaction on the internet can save a lot of human resources and material resources than the traditional negotiation and transaction. Especially automatic negotiation, which does not need a fixed place to negotiate with many participators [1] [2], can reduce side effect of the traditional negotiation and transaction.

The task of negotiation can be delegated to a software agent [3][4] in order to save users' time on activities which are either routine or demanding in e-commerce [5]. Some experts predict that there will be paradigm shift in e-commerce due to the emergence of agent [6], and many people are focusing on agent in recent years. [7] There are bilateral and multi-lateral negotiations according to the number of negotiators; there are single issue and multi-issue negotiations according to the number of issues concerned in negotiations. Here we focus on the bilateral multiissue negotiation, because it is more difficult than bilateral single issue negotiation and it is a basic tool for multi-lateral multi-issue negotiation.

But due to complexity of e-commerce negotiation's and negotiation's processing is difficult to control, common negotiation model is difficult to be

Please use the following format when citing this chapter:

Zhai, D., Wu, Y., Lu, J., Yan, F., 2007, in IFIP International Federation for Information Processing, Volume 251, Integration and Innovation Oricnt to E-Socicty Volumc1, Wang, W. (Eds), (Boston: Springcr), pp. 35-43. 
implemented. There are many works on theory of automatic negotiation of ecommerce [8][9], but there are few works on programming of automatic negotiation of e-commerce to apply in real e-commerce negotiation. [10][11]Many people are developing multi-agent system by genetic algorithms [1][3][5], but few are applying in automatic negotiation as a learning mechanism.

TOPSIS [12][ 13] (Technique for Order Preference by Similarity to Ideal Solution) is a classical multiple criteria decision making (MCDM) method. The negotiators can either accept or reject the offers depending on their utility [6][7]. If the negotiator's utility is higher than a specified value, he may accept the offer. If his utility is lower than the specified value, he may reject the offer. But there are many uncertain factors in negotiation. First, negotiators' preferences (or weights) are uncertain and dynamic. It is difficult to get exactly negotiators' preferences. Secondly, the evaluation of the solution is uncertain, which makes utility functions inaccurate. [14] So people always do not accept or refuse the offers definitely but at a certain degree in the business transaction. Considering these uncertain factors, we should measure the degree of acceptance or rejection of the negotiators for the offer by fuzzy numbers [13]. Therefore we evaluate the acceptability by membership function based on the fuzzy set theory [15][16].

There are various learning mechanisms in automatic negotiations such as Bayesian learning, case based reasoning and genetic algorithms [3]. Genetic algorithms [1] are useful for optimization and Rubenstein Montano and Malaga [17] states a genetic algorithms negotiation mechanism for searching optimal solutions for multiparty multi-objective negotiations. Genetic algorithms based on negotiation agents were used in the dynamic concession of bi-lateral negotiation [18]. Genetic algorithms are inspired by natural evolution, i.e. selection, reproduction, crossover and mutation. The basic principles of selection are means to select an optimal from initial populations of feasible solutions for a fitness function. Genetic algorithms for negotiation denote a set of feasible offers of the negotiation agent as a population of chromosomes. The fitness of a chromosome or a feasible offer is evaluated through the fitness function.

First, we provide a fuzzy negotiation model of e-commerce, which we state in our paper [19]. Secondly, we give a better fuzzy negotiation with genetic learning mechanism. Thirdly, we simulate the fuzzy negotiation model by genetic algorithms.

\section{A Fuzzy Negotiation Model of E-commerce with Genetic Algorithms}

Here we adopt the monotonic concession protocols as our negotiation protocols. [20] Agents start by assigning certain initial solutions from the feasible solution space to the negotiation variables [21]. An agreement is reached if one agent matches (or exceeds) what the other one asked for; otherwise the negotiation proceeds to the next step. The negotiation continues to another round if neither agent matches or exceeds the other's expectation. An agent is not allowed to offer the other agent lower prices than it did in the previous round, and it means that the agent has to increase at least one issue at one time as a counteroffer. If neither agent concedes at a 
certain step, then the negotiation ends with either an agreement or a deadlock. [22][23]

The advantage of the negotiation using the monotonic concession protocols is that it is similar to the real negotiation process, and it guarantees rapid convergence or stops the negotiation promptly when it is not convergent. Here we set a critical value $\lambda$ to decide if an offer is acceptable to the negotiator, i.e. the acceptability of the negotiator.

The offer from one agent is a vector $\left(x_{1}, x_{2}, \cdots, x_{n}\right)$, where ${ }^{x_{i}}(i=1, \ldots, \mathrm{n})$ is the value for the $i$ issue of the negotiation. Its membership function $v\left(x_{i}\right)$ is the acceptability for the value ${ }^{x_{i}}$, so the agent's offer can be represented as a

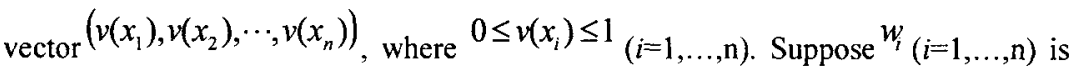
the weight for $i$ issue of the negotiation, the relative importance of $i$ issue for the negotiator, where $\sum_{i=1}^{n} w_{i}=1$, the negotiator's acceptability for the offer is represented as $\sum_{i=1}^{n} w_{i} v\left(x_{i}\right)$

We take normal membership function as the acceptability for both seller agent and buyer agent, $v(x)=e^{-\left(\frac{x-\infty b^{b}}{b}\right.}$ where $a>0, b>0$. Suppose the membership function of price is $v(x)=e^{-\left(\frac{x-a)^{2}}{b}\right.}$, where $\mathrm{a}>0, \mathrm{~b}>0, a$ is the negotiator's most acceptable price, which can be its initial offer, and $b$ is the negotiator's sensitivity for price.

When $b$ 's value is smaller, the sensitivity is smaller. The negotiator's acceptability is less sensitive to the price with smaller $b$ 's value when price varies in a bounded area; and vice versa. Suppose membership functions of the buyer and the seller both are normal distribution for convenience, and the left side is for the buyer and the right side is for the seller.

We set a critical value $\lambda$. When both negotiators' acceptability for the agent's offer exceeds or equals the critical value, the negotiation ends with a solution; otherwise the negotiator will exit the negotiation without a solution. We can set a critical value for the offer too. When the acceptability of both negotiators reaches the critical value, the negotiation can make an agreement.

Genetic algorithms are inspired by natural evolution, i.e. selection, reproduction, crossover and mutation. The basic principles of selection are means to select an optimal from initial populations of feasible solutions for a fitness function. Genetic algorithms for negotiation denote a set of feasible offers of the negotiation agent as a population of chromosomes. The fitness of a chromosome or a feasible offer is evaluated through the fitness function, and it is a combination of both seller's and buyer's acceptability. The fittest chromosome is a tentative solution for next round negotiation or a new offer.

The fitness function is a combination of both seller's and buyer's acceptability, the negotiation ends when both negotiators' acceptability reaches their target values 
rather than setting the iterative times. The genetic algorithms are as follows and flow chart of genetic algorithms is shown in Figure 1. choose an initial populationdetermine the fitness of each individualperform selection repeat perform crossover perform mutation determine the fitness of each individual perform selection until some stopping criterion applies.

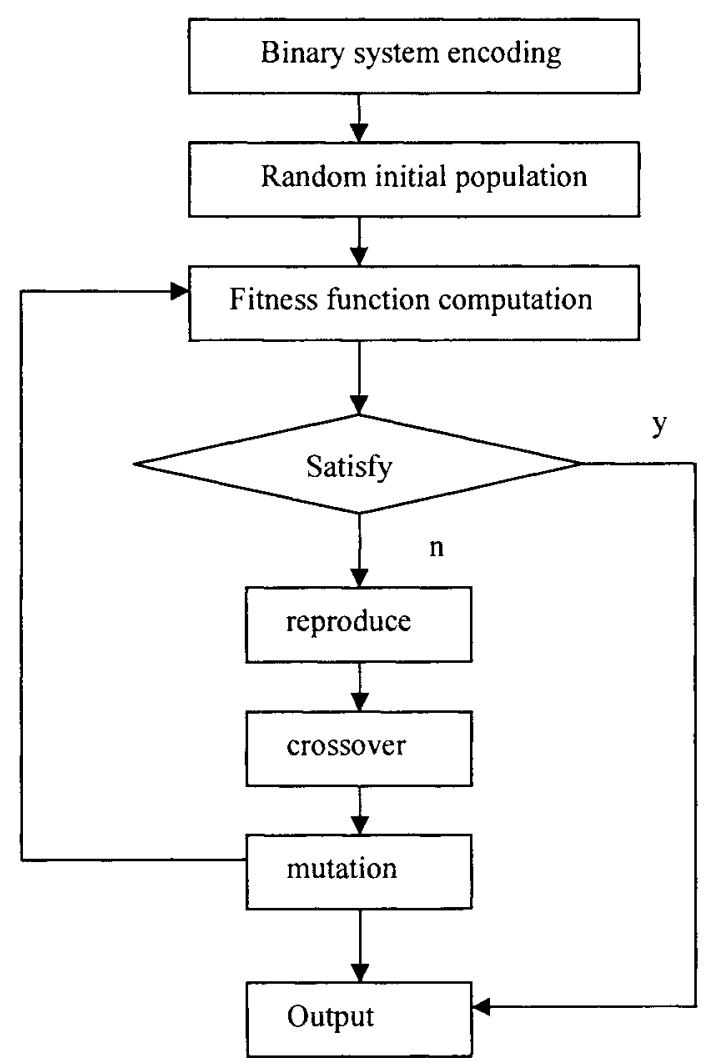

Fig 1 Flow chart of genetic algorithms

\section{An Example}

In the e-commerce, the buyer and the seller always negotiate about goods or service. Here we give a simple example to demonstrate the process of negotiation.

Suppose both the buyer and seller negotiate about four issues of goods, i.e. price, quality, transportation and service. Suppose the price for goods ranges from $¥ 10$ to $¥ 14$ per item, and the quality, transport and service are rated into 5 levels in Table 1 Negotiation issues.

Table 1 Negotiation issues 


\begin{tabular}{|llll|}
\hline \multicolumn{4}{|c|}{ Price C Quality } \\
\hline 10 & 4 & 4 & Transportation T Service S \\
11 & 3 & 3 & 3 \\
12 & 2 & 2 & 2 \\
13 & 1 & 1 & 1 \\
14 & 0 & 0 & 0 \\
\hline
\end{tabular}

Suppose the membership function of each issue is normal distribution, and the weight of each issue is the same. They are $\omega_{c}=0.6, \omega_{q=0.2}, \omega_{t=0.1}, \omega_{s=0.1}$ respectively.

We know price is the key issue for both buyer and seller. We set the critical values of both buyer and seller for price is 0.6 and the sensitivity for each issue is 3 .

Buyer's membership function for all issues are as follows: membership function for price is $v(x)=e^{-\left(\frac{x-10}{3}\right)^{2}}$; membership function for quality is $v(x)=e^{-\left(\frac{x-4}{3}\right)^{2}}$; membership function for transportation is $v(x)=e^{-\left(\frac{x-4}{3}\right)^{2}}$; membership function for service is $v(x)=e^{-\left(\frac{x-4}{3}\right)^{2}}$.

Seller's membership function for all issues are as follows: membership function for price is $v(x)=e^{-\left(\frac{x-14}{3}\right)^{2}}$; membership function for quality is $v(x)=e^{-\left(\frac{x}{3}\right)^{2}}$; membership function for transportation is $v(x)=e^{-\left(\frac{x}{3}\right)^{2}} ;$ membership function for service is $v(x)=e^{-\left(\frac{x}{3}\right)^{2}}$ 。

When acceptability of both buyer and seller for counteroffer exceeds $\lambda=0.6$, the offer is an acceptable offer. Suppose the sensitivity of both buyer and seller for time is the same and if one negotiator does not accept the counteroffer, he will reduce the acceptability for his own offer by $10 \%$. Suppose the initial offer of buyer is $(10,4,4,4)$ and the initial offer of seller is $(14,0,0,0)$.

In the end, we can get the offer $(12,2,2,3)$ as a solution. The membership functions of buyer and seller for the offer both reach 0.6 . The buyer's acceptability is $0.6 * 0.641+0.2 * 0.0 .641+0.1 * 0.641+0.1 * 0.895=0.6664$ and the seller's acceptability is $0.6 * 0.641+0.2 * 0.641+0.1 * 0.641+0.1 * 0.368=0.6137$. (Table 2 and Table 3 )

Table2 Seller's offer

\begin{tabular}{|c|c|c|}
\hline membership & seller & buyer \\
\hline seller's offer & & \\
\hline$(14,0,0,0)$ & 1 & 0.189 \\
\hline
\end{tabular}




\begin{tabular}{|l|l|l|}
\hline$(13,1,1,1)$ & 0.895 & 0.368 \\
\hline$(12,1,1,1)$ & 0.743 & 0.532 \\
\hline
\end{tabular}

Table3 Buyer's offer

\begin{tabular}{|c|c|c|}
\hline $\begin{array}{c}\text { membership } \\
\text { buyer's offer }\end{array}$ & seller & buyer \\
\hline$(10,4,4,4)$ & 0.189 & 1 \\
\hline$(11,3,3,3)$ & 0.368 & 0.895 \\
\hline$(12,3,3,3)$ & 0.532 & 0.743 \\
\hline$(12,2,2,3)$ & 0.666 & 0.614 \\
\hline
\end{tabular}

We can see the buyer and seller reach an agreement at the offer $(12,2,2,3)$. We know the fuzzy negotiation model is similar to the practical negotiation and the process of negotiation is similar too.

\section{Genetic Algorithms Parameters}

There are four issues to negotiate in the example, so we take four sections of genes to represent four items price, quality, transportation and service, their codes are shown in Figure 2. The difference between maxima and minima for each issue equals 4 , at least three bits are needed in binary code for each issue, so the coding length for four issues is 12 . Suppose the population size is 20 , probability of crossover is 0.95 and probability of mutation is 0.05 .
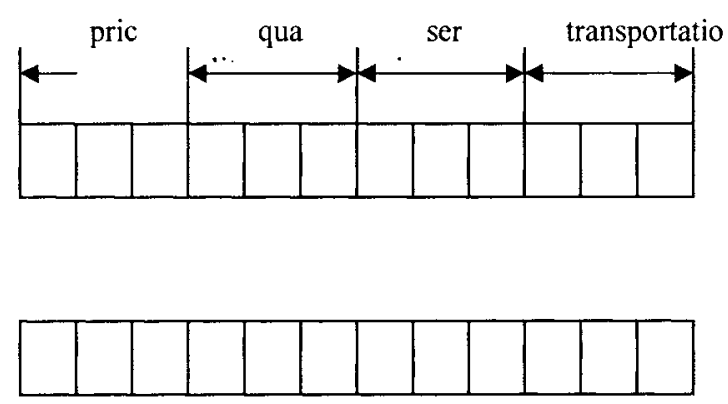

Fig 2 Binary code of negotiation

The initial offer or the first generation population of chromosomes is generated randomly, so simulations of negotiation process will change randomly. The final negotiation round of one simulation may differ from that of another simulation greatly, but the final offers of buyer agent and seller agent, and the acceptability of buyer agent and seller agent vary a little when buyer agent and seller agent reach an agreement, if we suppose that the ending condition for negotiation is both negotiators' acceptability reaches their target values. The final negotiation round of simulations may vary a little, but the final offers of buyer agent and seller agent, and the acceptability of buyer agent and seller agent in one simulation may differ from that in another simulation greatly, if we set the iterative times. The simulation result 
with target acceptability value is better than that with preset iterative times, and it is more practical.

The fitness function is a combination of both seller's and buyer's acceptability, the negotiation ends when both negotiators' acceptability reaches their target values rather than setting the iterative times. The fitness function can take various forms, and the fitness function of simulations is seller's acceptability multiplied by buyer's acceptability.

\section{Simulation}

The initial offers or the first generation population of chromosomes are generated randomly, so one simulation with the same preset acceptability may differ from another simulation greatly in iterative times, but final offers of buyer agent and seller agent and final acceptability of buyer agent and seller agent will vary within preset errors when they reach an agreement, because the ending condition of negotiation process is both negotiators' acceptability reaches their target values. The negotiation result is insensitive to the selection of first generation population and it is an advantage of genetic algorithms. One simulation output of negotiation is shown in Figure 3, the negotiation ends in 107 round. The final offer is $(12.857,4.0,4.0$, 2.286 ), seller agent's acceptability is 0.6256 and buyer agent's acceptability of buyer is 0.6144 . From the simulation result, both negotiators are satisfied with the final result, and genetic algorithms are practical learning mechanism in bilateral negotiations.

100111111101 offer $(12,286,4,0,4.0,2,057)$ accepability of seller $=0.5239295396955331$ accepability of buyer $=0.7222629148316078$ 10011111101 offer $(12,286,4,0,4,0,2,857)$ accepalbility of selier $=0.523929539595533$ t accepabilicy of buger $=0.7222629148316079$ 100111111101 offer $\{12.285,4.0,4.0,2.857\}$ accepability of seller $=0.5239295396955331$ accepability of buyer 20.7222629148316079 10011111101 offer $(12.285,4.0,4.0,2.85 ?)$ accepability of sel1er $* 0.5239295395955331$ accepability of buyer $=0.7222629148316078$ 100111111101 offer $(12.286,4.0,4.0,2.857)$ accepability of seller $=0.5239295396955331$ accepability of buger $=0.7222629148316078$

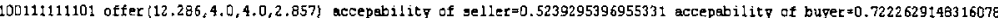
100111111101 offer $(12.286,4.0,4.0,2.857)$ accepability of seller 0.523929539695533 accepability of buyer $=0.7222629148316078$ 10011111101 offer $(12.285,4.0,4.0,2.857)$ accepanil1ty of ge11er $=0.5239295396955331$ accepab11ity of buger $=0.7222629148316078$ 100111111101 offer $(12.286,4.0,4.0,2.857)$ accepability of selier $=0.5239295396955331$ accepability of buper $=0.7222629148916078$ 10011111101 ofter $(12.285,9.0,4.0,2.857)$ accepability of geller $=0.5239295396955331$ accepability of buger $=0.7222629148316078$ 100111111101 offer $(12.286,4,0,4,0,2.857)$ accepability of seller $=0.5239295396955331$ accepability of buyer $=0.7222629148316078$ 100111111101 offer $(12.286,4.0,4.0,2.857)$ accepability of geller $=0.5239295396955331$ accepability of buger $\times 0.7222629148316078$ 107 's ehronosome

101111111100 offer $(12.857,4.0,4.0,2.286)$ accepab111tg of sel1er $=0,6255145571497915$ accepabil1ty of buper $=0.6143752542006342$ 101111111100 offer $(12.857,4.0,4.0,2.286)$ accepability of seller $=0.6256145571497915$ accepability of buppet $=0.6143752542 .006342$ 101111111100 offer $(12.857,4.0,4.0,2.286)$ accepability of gebier $=0.6256145571497915$ accepability of buper $=0.6143752542006342$ 101111111100 offer $(12.657,4,0,4,0,2.286)$ accepability of selier $=0.6256145571497915$ accepability of butgec $=0.6143752542006342$ 10111111100 ofter $(12,857,4.0,4,0,2.286)$ accerability of selier $=0.6256145571497915$ accepability of buper $=0.6143752542006342$ 10111111100 offer $\{12.857,4.0,4.0,2.286\}$ accepability of seller $=0.6256145571497915$ accepability of buper $=0.6143752542006342$ ID1111111100 ofter $(12.857,4.0,4.0,2.286)$ accepability of se12er $=0.6256145571497915$ accepability of buper 0.6143752542006392 10111111100 offer $(12.857,4,0,4,0,2.286)$ accepability of seller $=0.6256145571497915$ accepability of buper $=0.6143752542006342$ 101111111100 ofter $(12.057,4.0,4.0,2.286)$ accepability of seller $=0.6256145571497915$ accepabilicy of buter $=0.6143752542005342$ 101111111100 offer $(12.857,4.0,4.0,2.286)$ accepability of geller $=0.6256145571497915$ accepability of buper 40.6143752542006342 101111111100 offer $(12.857,4.0,4.0,2.286)$ accepability of ge 1 iar $=0.6256145571497915$ accepability of buper $=0.6143752542006342$ 101111111100 offer $(12.857,4.0,4.0,2.286)$ accepability of seller $=0.6256145571497915$ accepability of buper $=0.6143752542006342$ 10111111100 offer $\{12.857,4.0,4.0,2,286\}$ accepability of geller $=0.6256145571497915$ accepability of buper $=0,6143752542006342$ 101111111100 offer $(12.857,4,0,4,0,2.286)$ accepability of geller $=0.6256145571497915$ accepability of buper $=0.6143752542006342$ 101111111100 offer $(12.857,4.0,4.0,2.286)$ accepability of geller $=0.6256145571497915$ accepability of buper $=0.6143752542006342$ 10111111100 offer $(12.857,4.0,4.0,2.286)$ accepability of seller $=0.6255145571497915$ accepability of buper $=0.6143752542006342$ 101111111100 ofter $(12.857,4.0,4.0,2.286)$ accepability of gellex $=0.6256145571497915$ accepability of buppec $=0.6143752542006342$ 101111111100 offer $(12.957,4.0,4.0,2.285)$ accepability of seller $* 0.5256145571497915$ accepability of buper 20.6143752542006343 101111111100 offer $(12.857,4.0,4.0,2.286)$ accepability of se 1 ler $=0.5255145571497915$ accepasillity of buper $=0.6143752542006342$ io1111111100 offer(12.857,4.0,4.0,2.286) accepability of seller-0.6256145571497915 accepability of buper $=0.6143752542006342$

Fig 3 Output of genetic algorithms 


\section{Conclusions}

In traditional E-commerce negotiation, both negotiators always accept or reject an offer by a specified value. We give the fuzzy negotiation model based on genetic learning mechanism and evaluate the acceptability based on fuzzy set theory and membership functions so that the automatic negotiation is similar to the practical situation of negotiation. We adopt the monotonic concession protocol as our negotiation protocol and state the combined concession in the multi-issues negotiation for the negotiators and adopt genetic learning mechanism so that its negotiation outcome is more exact. This protocol is simple, so it is easy to implement automatically. The example shows the fuzzy negotiation model is reasonable and feasible and it is similar to the real negotiation process. The fuzzy negotiation model used here is the normal distribution. Other more complex distribution need further study. We hope that fuzzy multi-issue negotiation model of e-commerce can be applied in real business transaction.

\section{References}

I Samuel P.M.Choi, Jiming Liu, Sheung-Ping Chan. "A Genetic Agent-based Negotiation System". Computer Networks 37, 2001, 195-204.

2 Ren-Jye Dzeng, Yu-Chun Lin. "Intelligent Agents for Supporting Construction Procurement Negotiation". Expert Systems with Applications 27,2004, 107-1 19

3 Raymond Y.K. Lau, Towards Genetically Optimised Multi-Agent Multi-Issue Negotiations, Proceedings of the 38th Hawaii International Conference on System Sciences, $2005,1-10$

4 F. Bergenti, A. Poggi, An Agent-based Approach to Manage Negotiation Protocols in Rexible CSCW Systems, Proceeding of.4th International Conference on Autonomous Agents, Barcelona Spain, 2000, 267-268.

5 Raymond Y.K. Lau, Maolin Tang, On Wong, Towards Genetically Optimised Responsive Negotiation Agents, Proceedings of the IEEE/WIC/ACM International Conference on Intelligent Agent Technology (IAT'04), 2004, 1-7

6 W. C. Stirling. Social Utility Functions-Part 1: Theory, IEEE Transaction Systems, Man, Cybernetics. C, Applications and Reviews, vol. 35, no. 4, 2005 522-532

7 W. C. Stirling, R.L. Frost . Social Utility Functions-Part 2: Theory, IEEE Transaction Systems, Man, Cybernetics. C, Applications and Reviews, vol. 35, no. 4, 2005 533-543

8 Y.Y Wu, J.X Lu, "A Fuzzy Negotiation Model of E-commerce", Journal of System Science and Information, 2006, Vol.4, No.1, 33-37

9 S.L Zhao, G.R Jiang, T.Y Huang. An Analysis Model to Agent Ability Report Deception in Multi-agent Cooperation, Proceedings of 2005 International Conference on Management Science \& Engineering, 2005

10 S.L. Zhao, G.R Jiang, T.Y Huang. The Deception Detection and Restraint in Multiagent System, Proceedings of 17 th IEEE International Conference on Tools with Artificial Intelligence, 2005

11 D.S Zhai, J.X Lu, Y.Y Wu, "A Program of Automatic Negotiation in E-commerce", Journal of Information and Decision Science, 2006, Vol.1, No.1, 7-11 
12 Sheng-Hshiung Tsaur, T.Y Chang and C.H Yen, "The Evaluation of Airline Service Quality by Fuzzy MCDM", Tourism Management, 2002 (23), pp.107-115.

13 M. Bo, F. Wei, A Negotiation Model Based on Fuzzy Multiple Criteria Decision Making Method, Proceedings of the Fourth International Conference on Computer and Information Technology, CIT'04, 2004, $1039-1044$

14 C.-B. Cheng, C.-C. H. Chan, and K.-C. Lin, Intelligent agents for e-Marketplace: Negotiation with Issue Trade-offs by Fuzzy Inference Systems, Decision Support System.

15 Y.X Meng, B. Meng. An Agent-based Negotiation Support System with Fuzzy Multiobjective Decision-making Method, Proceedings of ICSSSM '05. 2005 International Conference on Services Systems and Services Management, 2005, 1141 - 1144 Vol. 2

16 Lai. K.R. Chung Hsien Lan. Development of an Assessment Agent to Promote the Learning Effectiveness in a Computer Supported Collaborative Learning Environment, Proceeding of Fifth IEEE International Conference on Advanced Learning Technologies, 2005. ICALT 2005. $354-358$

17 Bonnie Rubenstein-Montano, Ross A. Malaga, A Weighted Sum Genetic Algorithm to Support Multiple-Party Multiple-Objective Negotiations, IEEE Transactions on Evolutionary Computation, Vol. 6, No. 4, 2002, 366-377

18 Ravindra Krovi, Arthur C. Graesser, William E. Pracht, Agent Behaviors in Virtual Negotiation Environments, IEEE Transactions on Systems, "Man, and Cybernetics-Part C": Applications and Reviews, Vol. 29, No.1, 1999, 15-25

19 Y.Y Wu, J. Lu, F. Yan, A Fuzzy Negotiation Model of e-Commerce and Its Implementation, Technology Management for the Global Future, PICMET 2006 Proceedings, Vol.3, 9-13 July, Istanbul, Turkey, 1180-1185

20 Z.Ren*,C.J.Anumba,O.O.Ugwu. The Development of a Multi-agent System for Construction Claims Negotiation. Advances in Engineering Software 34,2003, 683-696.

21 Wei-Po Lee. Towards Agent-based Decision Making in the Electronic Marketplace: Interactive Recommendation and Automated Negotiation. Expert Systems with Applications $27,2004$.

22 Chun, A., Wai, H., \& Wong, R.. Optimizing Agent-based Meeting Scheduling through Preference Estimation. Engineering Applications of Artificial Intelligence, 16, 727-743

23 Chang-Shing Lee,Chen-Yu Pan. An Intelligent Fuzzy Agent for Meeting Scheduling Decision Support System. Fuzzy Sets and Systems 142,2004, 467-488 